\title{
Effect of cocaine on periadolescent rats with or without early maternal separation
}

C.S. Planeta and M.T. Marin
Laboratório de Farmacologia, Faculdade de Ciências Farmacêuticas,

Universidade Estadual Paulista, Araraquara, SP, Brasil

\section{Correspondence \\ C.S. Planeta \\ Laboratório de Farmacologia \\ FCF, UNESP \\ Rod. Araraquara-Jaú, km 1 \\ 14801-902 Araraquara, SP \\ Brasil \\ Fax: +55-16-222-0073 \\ E-mail: cplaneta@fcfar.unesp.br}

Research supported by FAPESP

(Nos. 97/11010-2 and 00/09319-0).

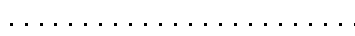

Received February 25, 2002

Accepted August 22, 2002

\begin{abstract}
Cocaine-induced behavioral sensitization and weight loss were investigated in periadolescent Wistar rats kept with their mothers or subjected to repeated maternal separation. Litters allocated to the separation procedure were placed in a temperature-controlled $\left(33^{\circ} \mathrm{C}\right)$ chamber for $3 \mathrm{~h}$ per day from postnatal day 6 (P6) to P20. Non-handled rats were left undisturbed until weaning. Treatments were started on P3031 and the test was performed on P36-37. Animals received injections of saline or cocaine $(10 \mathrm{mg} / \mathrm{kg}, s c)$ twice daily for 5 days. On day 6 all animals received saline. On day 7 animals were challenged with 10 $\mathrm{mg} / \mathrm{kg}$ cocaine and their locomotion was evaluated in activity cages. A third group received saline throughout the 7-day period. Body weights were recorded on P30-31 and P36-37. Two-way ANOVA on body weights showed a main effect of treatment group $(F(1,35)=10.446$, $\mathrm{P}=0.003 ; \mathrm{N}=10-12)$. Non-handled rats treated with cocaine for 5 days gained significantly less weight, while no significant effect was observed in maternally separated rats. Two-way ANOVA revealed a main effect of drug treatment on locomotor activity $(\mathrm{F}(2,32)=15.209$, $\mathrm{P}<0.001 ; \mathrm{N}=6-8)$, but not on rearing condition $(\mathrm{F}(1,32)<0.001$, $\mathrm{P}=0.998$ ). Animals pretreated with cocaine showed a clear behavioral sensitization relative to the saline group. No difference in the magnitude of sensitization was found between separated and non-handled animals. Only the effect of cocaine on weight gain was significantly affected by repeated episodes of early maternal separation during the pre-weaning period.
\end{abstract}

Adolescents across a variety of species exhibit age-specific behaviors that can help them attain the necessary skills for adult independence. These adolescent-related characteristics, such as risk taking and novelty seeking, may be promoted by developmental events in the central nervous system and can predispose adolescents to initiating drug use (1).

Behavioral sensitization is a progressive and enduring enhancement of the motor stimulant effects of cocaine and other drugs of abuse, elicited by repeated administration of these drugs (2). Behavioral sensitization has been extensively studied in adult animals because it is believed to be a key component of the drug addiction process (3). Comparably few studies have assessed behavioral sensitization in adolescent animals, although this type of study is potentially important for early human psychostimulant use since drug abuse among humans often begins during the 
adolescence period (4).

It has been extensively demonstrated that stressful experiences in adulthood have a strong influence on susceptibility to drugtaking behavior $(5,6)$. Moreover, there is evidence that an animal's response to a drug can be affected by early exposure to stress. For example, maternal separation during the pre-weaning period leads to altered behavioral responses to cocaine and amphetamines in adult animals (7-9). Thus far, investigations addressing the interplay between stress and the effects of drugs of abuse have been devoted mainly to adult subjects.

Many adolescent-specific neurobehavioral alterations observed in humans are also seen in rats of comparable age. Spear and Brake (10) defined periadolescence as the age period around the time of sexual maturation when age-specific behavioral and psychopharmacological discontinuities are evident. Using this criterion, postnatal days 3042 were designated as periadolescence in rats (10).

We investigated cocaine-induced behavioral sensitization in periadolescent rats, and how it is affected by maternal separation. The effect of cocaine on body weight was also monitored.

Primiparous pregnant Wistar dams were obtained from the animal breeding facility of the Institute of Biomedical Science, University of São Paulo, SP, Brazil. The date of birth was defined as postnatal day 0 (P0). On P1 litters were culled to 7-11 pups. Dams and their pups were housed together in plastic cages measuring 40 (length) $\times 32$ (width) x 16 (height) $\mathrm{cm}$. All animals were kept in the same temperature- and humidity-controlled holding facility $\left(22 \pm 2^{\circ} \mathrm{C}\right)$ on a $12 /$ 12-h light/dark cycle (lights on at 7:00 am) with food and water available ad libitum.

The litters allocated to the separation procedure were removed from their nest cages and placed in small plastic cages in a temperature-controlled $\left(33^{\circ} \mathrm{C}\right)$ chamber for $3 \mathrm{~h}$ per day from P6 to P20. Pup weight was re- corded on P7, P13 and P21. Control rat litters (non-handled), originating from litters born in the colony at about the same time, were left undisturbed until weaning, except for cleaning the cages and weighing on P7, $\mathrm{P} 13$ and P21.

After weaning, on P21, the siblings were separated by gender and only males were housed in groups of four in standard cages. No more than two individuals per litter were used for any given test condition. The animals were allowed free access to food and water, except during observation.

The dose of cocaine used in these experiments is known to induce locomotor activity in the absence of focused stereotypies (11). The schedule for repeated cocaine exposure was adapted from Laviola et al. (12), who studied cocaine sensitization in periadolescent and adult rats.

The entire sensitization protocol took 7 days. On days 1 to 5 , the rats were weighed and given a subcutaneous injection of 10 $\mathrm{mg} / \mathrm{kg}$ cocaine (COC) hydrochloride (Sigma, St. Louis, MO, USA) or saline (SAL) twice a day (7:00 am and 7:00 pm). Immediately after injection, the animals were returned to their home cages.

On day 6 all animals received saline. On day 7 (test) both SAL and COC pretreatment groups received a challenge dose of $10 \mathrm{mg} / \mathrm{kg}$ cocaine ( $\mathrm{SAL}+\mathrm{COC}$ and $\mathrm{COC}+\mathrm{COC}$ groups). Immediately after the injections their locomotor activity was recorded in commercially available (Columbus Instruments, Columbus, $\mathrm{OH}, \mathrm{USA}$ ) activity monitoring chambers consisting of Plexiglas cages equipped with 10 matched pairs of photocells which were used to measure the horizontal locomotor activity. The consecutive interruption of two beams was recorded as one locomotion unit. Photocell counts accumulated during 5-min periods were recorded during a 45min testing session. The animals were allowed a 30-min adaptation period in the photocell apparatus prior to injection. A third group of animals received saline injections 
throughout the 7-day period (SAL + SAL).

Treatments started when the rats were at P30-31 and the test was performed on P3637. Body weights were also recorded on P30-31 and P36-37.

All data are reported as means \pm SEM. Behavioral data were analyzed using $3 \times 2$ (treatment group $\mathrm{x}$ rearing condition) ANOVA. Body weight gain during the sensitization protocol was analyzed by $2 \times 2$ (drug treatment $x$ rearing condition) ANOVA. Appropriate post-hoc comparisons were performed using the Newman-Keuls test.

Two-way ANOVA applied to body weight data showed a main effect of treatment group $(\mathrm{F}(1,35)=10.446 ; \mathrm{P}=0.003)$, but not of rearing condition $(\mathrm{F}(1,35)=0.066$; $\mathrm{P}=0.798)$. In addition, no significant interaction between factors was detected $(\mathrm{F}(1,35)$ $=0.517 ; \mathrm{P}=0.477)$. Pairwise comparison by the Newman-Keuls test revealed that nonhandled rats treated with cocaine for 5 days $(\mathrm{COC}+\mathrm{COC})$ had a significantly lower weight gain when compared to the SAL + SAL group $(\mathrm{P}<0.05)$, whereas the reduction in body weight gain in maternally separated rats was not significant $(\mathrm{P}=0.08)$. Therefore, as found with adult subjects, cocaine also makes periadolescent animals anorexic. However, this effect was not significant in rats separated from their mother at an early age (Figure 1), suggesting that maternal separation altered neural systems that regulate body weight. In fact, although no changes in body weight were observed during the preweaning period, the body weights were significantly higher in the maternally separated adolescent rats when compared to nonhandled ones (data not shown).

As indicated in Figure 2, two-way ANOVA revealed a major effect of drug treatment on locomotor activity $(\mathrm{F}(2,32)=$ $15.209 ; \mathrm{P}<0.001)$ but not on rearing condition $(\mathrm{F}(1,32)<0.001 ; \mathrm{P}=0.998)$. In addition, no interaction between factors was detected $(\mathrm{F}(2,32)=0.060 ; \mathrm{P}=0.942)$.

Post-hoc analyses did not show any sig-

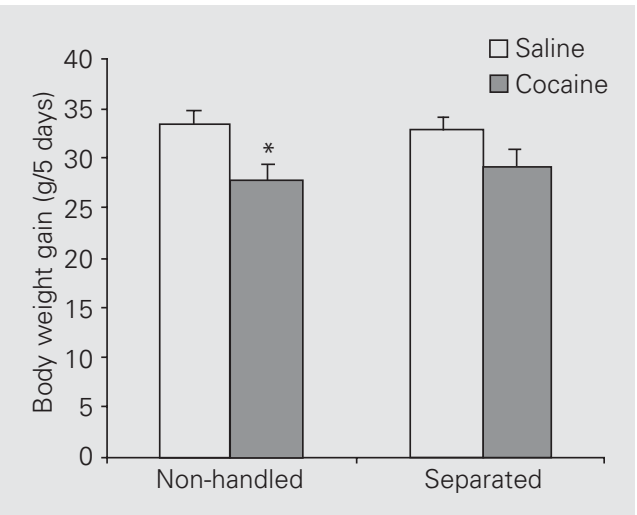

Figure 1. Effect of repeated cocaine injections on body weights of non-handled and early maternally separated periadolescent rats. The rats received twice daily injections of $10 \mathrm{mg} / \mathrm{kg}$ cocaine, Sc, for 5 days. Data are reported as means \pm SEM for 10-12 animals per group. ${ }^{*} \mathrm{P}<0.05$ compared to the respective saline control (Newman-Keuls test).
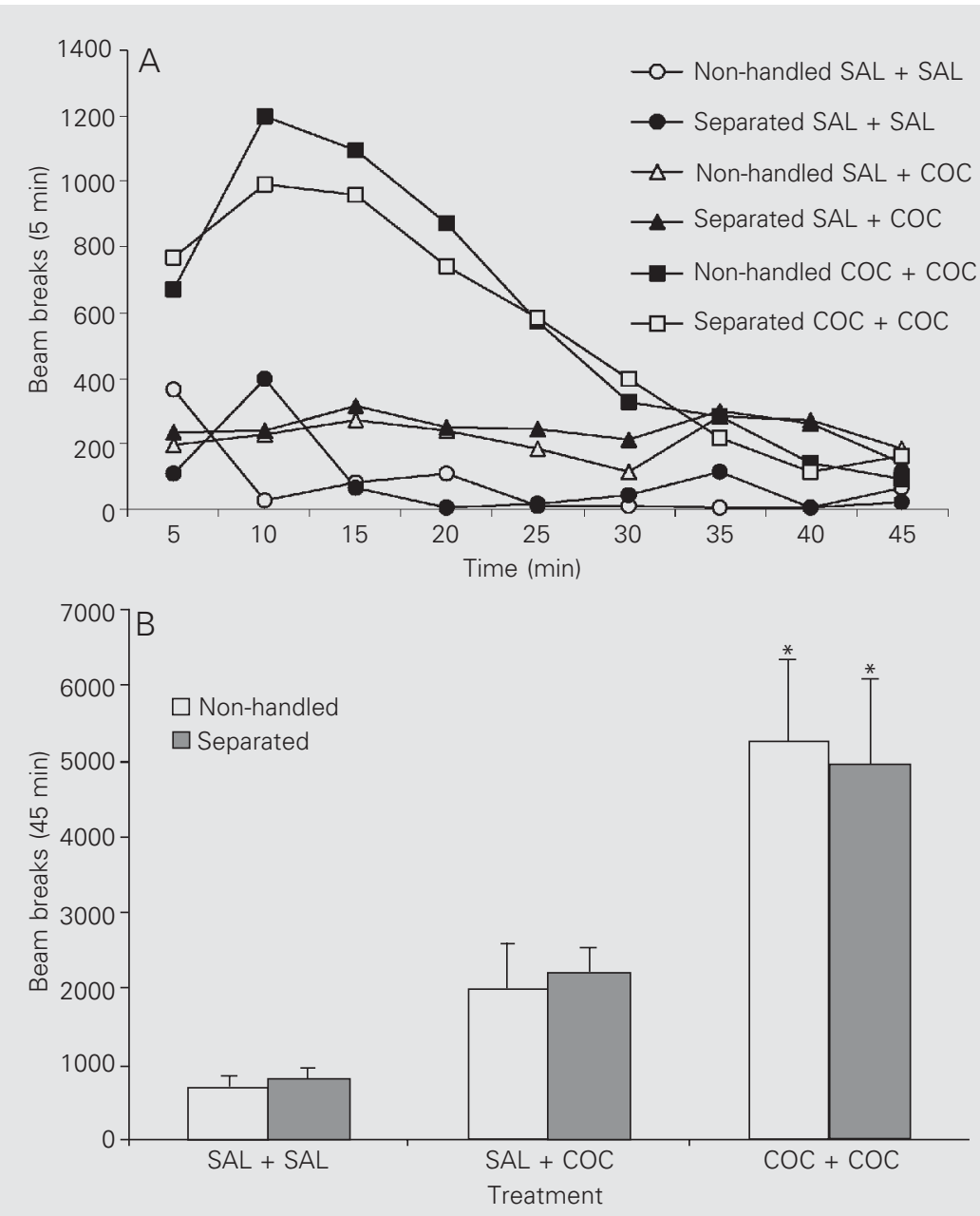

Figure 2. Effect of repeated cocaine injections on locomotor activity after a challenge dose of cocaine in non-handled and early maternally separated periadolescent rats. A challenge dose of $10 \mathrm{mg} / \mathrm{kg}$ cocaine ip was administered to animals pretreated with twice daily injections of $10 \mathrm{mg} / \mathrm{kg}$ cocaine $s c$ for 5 days. Data are reported as means \pm SEM for 6-8 animals per group of cumulative photocell counts during 5 -min (A) and 45-min intervals (B). ${ }^{*} \mathrm{P}<0.05$ compared to the respective SAL + SAL or SAL + COC control (Newman-Keuls test). $\mathrm{SAL}=$ saline, $\mathrm{COC}=$ cocaine. 
nificant difference in locomotor activity when comparing the SAL + COC to SAL + SAL groups in either non-handled or separated rats. However, locomotion was significantly higher in the $\mathrm{COC}+\mathrm{COC}$ compared to both the SAL + SAL and the SAL + COC groups $(\mathrm{P}<0.05)$. Thus, repeated exposure to cocaine induces behavioral sensitization in periadolescent rats, which is not affected by maternal separation.

Administration of cocaine $(10 \mathrm{mg} / \mathrm{kg})$ to animals pretreated with saline produced a nonsignificant increase in locomotor activity. In another experiment we found a significant increase in locomotion in adult rats injected with the same dose of cocaine (13).

The finding that adolescent rats are less sensitive to the acute effects of cocaine is consistent with previous reports which indicated that periadolescents have a characteristic hyporesponsiveness to the effects of acute administration of psychostimulants on locomotor activity (14). Periadolescent rats have also been found to have a reduced sensitivity to amphetamine-induced place preference (15), as well as a marked resistance to amphetamine-induced taste aversion (16). According to Ramsay and Woods (17), the initial insensitivity to psychoactive drugs might underlie an increased risk for addiction. This lower sensitivity might lead to repeated experiences with the drug to achieve its rewarding effects, and could provoke sensitization, which is strongly linked to the development of drug dependence. In fact, despite the low level of locomotor activation after acute cocaine exposure, adolescent rats treated with cocaine for 5 days showed a marked increase in locomotion counts after a challenge dose of cocaine given $48 \mathrm{~h}$ after the last injection. Thus, the present study confirms the findings of Laviola et al. (12) who observed sensitization to the behavioral effects of cocaine in periadolescent rats. This finding is particularly interesting in view of the few studies in the literature on the ontogenetic characteriza- tion of psychostimulant-induced behavioral sensitization.

There is evidence that pre- as well as postsynaptic alterations in dopaminergic neurotransmission are relevant for the manifestation of sensitization (3). Laviola et al. (18), using in vivo microdialysis, demonstrated that periadolescent rats treated with amphetamine show a strong sensitization of amphetamine-stimulated dopamine release, whereas no such change was observed in adult animals. Therefore, different mechanisms might underlie psychostimulant-induced behavioral sensitization in adults versus periadolescent animals.

A 3-h maternal separation from P6 to P20 had no affect on either acute or repeated effects of cocaine-induced locomotion in periadolescent rats.

Few studies have investigated the influence of maternal separation on the effects of psychostimulants. Matthews et al. (7) reported an attenuated locomotor response to amphetamines in adult female, but not male, rats that had been separated from their dams for $6 \mathrm{~h}$ on 10 occasions between P5 and P20.

The acquisition and maintenance of cocaine self-administration in adult rats separated from their dams during the pre-weaning period is gender and dose dependent. For example, a reduced response to cocaine was observed by Matthews et al. (8) at the 0.05 $\mathrm{mg} /$ injection dose only for female rats. Additionally, they found facilitated acquisition of cocaine self-administration at a higher dose $(0.08 \mathrm{mg} /$ injection $)$ in females that had been maternally separated (8).

Recently, Kosten et al. (9) reported that 1-h maternal isolation from P2-P9 enhances the acquisition of cocaine self-administration in adult male rats.

It is difficult to compare our results with the literature since: a) the nature of the physiological changes produced by maternal separation depends upon the specifics of the separation procedure, the environmental conditions, the postnatal period, and the dura- 
tion of separation, and b) so far, none of the studies have investigated the consequences of maternal separation on the effects of cocaine on adolescent rats. These facts point to the relevance of the present study on the influence of different periods of maternal separation on the effects of psychostimulants, especially in adolescent animals.

During late childhood and adolescence, neurobiological systems are still undergoing important developmental rearrangements. In addition, hormone levels change dramatically during adolescence as a result of the onset of puberty (for discussion, see Ref. 1). A better characterization of cocaine-induced behavioral sensitization in adolescents will require careful delineation of behavioral, neurochemical, neuroendocrine and neuroana- tomical aspects and their modification by environmental manipulation.

However, behavioral sensitization and the potential impact of environmental factors and drugs during adolescence have received surprisingly little attention. A better understanding of the effect of psychostimulants during adolescence and their modification by early stress should allow the development of more effective prevention and treatment strategies.

\section{Acknowledgments}

We thank Elisabete Zocal Paro Lepera and Rosana Pupim Silva for technical assistance and Celso Luís Borsato for animal care.

\section{References}

1. Spear $L$ (2000). The adolescent brain and age-related behavioral manifestations. Neuroscience and Biobehavioral Reviews, 24: 417-463

2. Robinson TE \& Becker JB (1986). Enduring changes in brain and behavior produced by chronic amphetamine administration: a review and evaluation of animal models of amphetamine psychosis. Brain Research, 396: 157-198.

3. Robinson TE \& Berridge KC (1993). The neural basis of drug craving: an incentive salience theory of addiction. Brain Research Reviews, 18: 247-291.

4. Anthony JC \& Petronis KR (1995). Earlyonset drug use and risk of later drug problems. Drug and Alcohol Dependence, 40: 9-15.

5. Erb S, Shaham Y \& Stewart J (1996). Stress reinstates cocaine-seeking behavior after prolonged extinction and a drugfree period. Psychopharmacology, 128: 408-412.

6. Miczek KA \& Mutschler NH (1996). Activational effects of social stress on iv cocaine self-administration in rats. Psychopharmacology, 128: 256-264.

7. Matthews K, Hall FS, Wilkinson LS \& Robbins TW (1996). Retarded acquisition and reduced expression of conditioned locomotor activity in rats following re- peated early maternal separation: effects of prefeeding, d-amphetamine, dopamine antagonists and clonidine. Psychopharmacology, 126: 75-84.

8. Matthews K, Robbins TW, Everitt BJ \& Caine SB (1999). Repeated neonatal maternal separation alters intravenous cocaine self-administration in adult rats. Psychopharmacology, 141: 123-134.

9. Kosten TA, Miserendino MJD \& Kehoe P (2000). Enhanced acquisition of cocaine self-administration in adult rats with neonatal isolation stress experience. Brain Research, 875: 44-50.

10. Spear LP \& Brake SC (1983). Periadolescence: age-dependent behavior and psychopharmacological responsivity in rats. Developmental Psychobiology, 16: 83109.

11. Kuczenski R, Segal DS \& Aizenstein ML (1991). Amphetamine, cocaine, and fencanfamine: relationship between locomotor and stereotypy response profiles and caudade and accumbens dopamine dynamics. Journal of Neuroscience, 11: 2703-2712.

12. Laviola G, Wood RD, Kuhn C, Francis R \& Spear $L$ (1995). Cocaine sensitization in periadolescent and adult rats. Journal of Pharmacology and Experimental Therapeutics, 275: 345-357.
13. Araujo APN, DeLucia R, Scavone C \& Planeta CS (2002). Repeated predictable or unpredictable stress: effects on cocaine effects and cyclic AMP-dependent protein kinase. Behavioural Brain Research (in press).

14. Adriani W \& Laviola G (2000). A unique hormonal and behavioral hyporesponsivity to both forced novelty and d-amphetamine in periadolescent mice. Neuropharmacology, 39: 334-346.

15. Adriani W, Chiarotti F \& Laviola G (1998). Elevated novelty seeking and typical damphetamine sensitization in periadolescent compared to adult mice. Behavioral Neuroscience, 112: 1152-1166.

16. Infurna RN \& Spear LP (1979). Developmental changes in amphetamine-induced taste aversions. Pharmacology, Biochemistry and Behavior, 11: 33-35.

17. Ramsay DS \& Woods SC (1997). Biological consequences of drug administration: implications for acute and chronic tolerance. Psychological Reviews, 104: 170193.

18. Laviola G, Pascucci T \& Pieretti S (2001). Striatal dopamine sensitization to d-amphetamine in periadolescent but not in adult rats. Pharmacology, Biochemistry and Behavior, 68: 115-124 